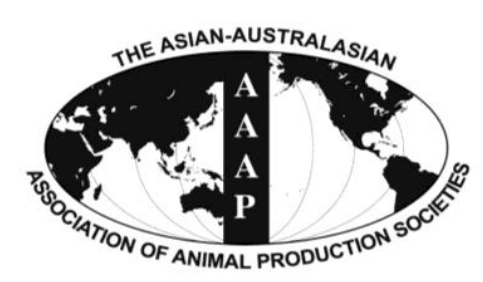

Open Access

Asian Australas. J. Anim. Sci.

Vol. 27, No. 12 : 1726-1735 December 2014

http://dx.doi.org/10.5713/ajas.2014.14292

www.ajas.info

pISSN 1011-2367 elSSN 1976-5517

\title{
Effects of Acarbose Addition on Ruminal Bacterial Microbiota, Lipopolysaccharide Levels and Fermentation Characteristics In vitro
}

\author{
Yu-yang Yin, Yu-jie Liu, Wei-yun Zhu, and Sheng-yong Mao* \\ College of Animal Science and Technology, Nanjing Agricultural University, Nanjing 210095, China
}

\begin{abstract}
This study investigated the effects of acarbose addition on changes in ruminal fermentation characteristics and the composition of the ruminal bacterial community in vitro using batch cultures. Rumen fluid was collected from the rumens of three cannulated Holstein cattle fed forage ad libitum that was supplemented with $6 \mathrm{~kg}$ of concentrate. The batch cultures consisted of $8 \mathrm{~mL}$ of strained rumen fluid in $40 \mathrm{~mL}$ of an anaerobic buffer containing $0.49 \mathrm{~g}$ of corn grain, $0.21 \mathrm{~g}$ of soybean meal, $0.15 \mathrm{~g}$ of alfalfa and $0.15 \mathrm{~g}$ of Leymus chinensis. Acarbose was added to incubation bottles to achieve final concentrations of $0.1,0.2$, and $0.4 \mathrm{mg} / \mathrm{mL}$. After incubation for $24 \mathrm{~h}$, the addition of acarbose linearly decreased $(\mathrm{p}<0.05)$ the total gas production and the concentrations of acetate, propionate, butyrate, total volatile fatty acids, lactate and lipopolysaccharide (LPS). It also linearly increased $(\mathrm{p}<0.05)$ the ratio of acetate to propionate, the concentrations of isovalerate, valerate and ammonia-nitrogen and the $\mathrm{pH}$ value compared with the control. Pyrosequencing of the 16S rRNA gene showed that the addition of acarbose decreased $(\mathrm{p}<0.05)$ the proportion of Firmicutes and Proteobacteria and increased $(\mathrm{p}<0.05)$ the percentage of Bacteroidetes, Fibrobacteres, and Synergistetes compared with the control. A principal coordinates analysis plot based on unweighted UniFrac values and molecular variance analysis revealed that the structure of the ruminal bacterial communities in the control was different to that of the ruminal microbiota in the acarbose group. In conclusion, acarbose addition can affect the composition of the ruminal microbial community and may be potentially useful for preventing the occurrence of ruminal acidosis and the accumulation of LPS in the rumen. (Key Words: Acarbose, Ruminal Microbiota, Pyrosequencing, In vitro Fermentation)
\end{abstract}

\section{INTRODUCTION}

The energy and fibre dietary requirements of highyielding dairy cows are not easily met. This is especially true for early lactation cows because the energy they expend exceeds the energy they consume. Thus, feeding highly fermentable diets to dairy cattle is a common strategy to increase energy intake. However, highly fermentable diets are rapidly converted to organic acids (i.e. short-chain fatty acids and lactic acid) in the rumen. The resulting release of protons can constitute a challenge to the ruminal ecosystem and animal health and lead to subacute ruminal acidosis (SARA) (Krause et al., 2006). Screening 15 Holstein herds in the US revealed the presence of SARA

\footnotetext{
* Corresponding Author: Sheng-yong Mao. Tel: +86-025-8439 5523, Fax: +86-025-84395314, E-mail: maoshengyong@163.com Submitted Apr. 22, 2014; Revised Jun. 24, 2014; Accepted Aug. 4, 2014
}

in $19 \%$ of early lactation cows and $26 \%$ of mid-lactation cows (Garrett et al., 1997). As SARA affects animal health and reduces milk yields, SARA is considered a major nutritional disorder in ruminants (Krause et al., 2006). In addition to compromising the health of dairy cows and increasing costs, SARA is of concern for animal welfare reasons, as ruminal acidosis can cause lameness and laminitis, both of which significantly affect the comfort and general well-being of the animal (Cook et al., 2004).

Lipopolysaccharide (LPS) is a component of the cell wall of gram-negative bacteria, which are the predominant bacterial group in the rumen. Previous studies revealed that a decline in ruminal $\mathrm{pH}$ during SARA causes the death and cell lysis of gram-negative bacteria, resulting in an increase in the free ruminal LPS concentration (Nagaraja et al., 1978; Nagaraja and Titgemeyer, 2007; Plaizier et al., 2008). A low ruminal $\mathrm{pH}$, together with high LPS levels during SARA, can reduce the barrier function of the epithelium of 
the rumen, potentially allowing LPS to translocate into blood and stimulate a systemic inflammatory response (Zebeli and Metzler-Zebeli, 2012). Therefore, it is very important to prevent the accumulation of LPS and to stabilise the ruminal $\mathrm{pH}$ during SARA.

Many strategies have been used to improve the ruminal $\mathrm{pH}$ during SARA. The use of ionophores (Packer et al., 2011) and probiotics based on yeasts, such as Saccharomyces cerevisiae (Desnoyers et al., 2009), have been found to stabilise the ruminal $\mathrm{pH}$ and to improve milk production. Recently, some new approaches for controlling the $\mathrm{pH}$, including $\alpha$-amylase, glucosidase inhibitors and organic acid, have been explored (McLaughlin et al., 2009a,b; Remling et al., 2013). Of them, the glucosidase inhibitors such as acarbose has been demonstrated to have beneficial effects in preventing the incidence of acidosis in steers (McLaughlin et al., 2009a), and in reversing the decrease in feed intake and milk fat when feeding dairy cattle a diet containing a high ratio of concentrate (McLaughlin et al., 2009b). However, it is not clear whether acarbose affects the concentration of ruminal LPS. In addition, although previous studies revealed that the addition of acarbose produces a lower Streptococcus bovis to Megasphaera elsdenii ratio than in the rumen of the control group (Blanch et al., 2010), little information is available on the effects of acarbose on the composition of the ruminal microbial community.

In this study, we hypothesised that acarbose affects the composition of the ruminal bacterial community and that it decreases the concentration of LPS in the rumen. Thus, this research investigated the effects of acarbose addition on changes in ruminal fermentation characteristics, the levels of LPS, and the composition of the ruminal microbial community in vitro.

\section{MATERIALS AND METHODS}

\section{Compounds and animal husbandry conditions}

Acarbose (Precose) (Bayer Corporation, West Haven, CT, USA) is a microbial-derived $\alpha$-glucosidase inhibitor, which is marketed as a treatment for human and animal type II (i.e. noninsulin-dependent) diabetes mellitus. Three ruminally cannulated Holstein cows $(500 \pm 23.5 \mathrm{~kg} \mathrm{BW})$ served as ruminal fluid donors for these experiments. The cattle were fed a diet of corn straw ad libitum, supplemented with $6 \mathrm{~kg}$ of concentrate. The ingredients of the concentrate included corn, soybean meal and wheat bran. The composition ( $\%$ dry matter [DM]) of the diet was crude protein 12.3 , neutral detergent fibre 40.4 and acid detergent fiber 17.2. The cattle were fed twice daily at 6.30 and 18.00 h. All animal care was conducted in accordance with procedures approved by the Animal Care and Use
Committee of Nanjing Agricultural University.

\section{Treatments and sampling}

The substrate (490 mg of ground corn kernels, $210 \mathrm{mg}$ of ground soymeal bean, $150 \mathrm{mg}$ of ground Leymus chinensis hay and $150 \mathrm{mg}$ of ground alfalfa hay, DM based) was oven dried, weighed and placed in bottles. The groups were divided into a control group ( $0 \mathrm{mg}$ acarbose) and three acarbose treatment groups, with 4,8 , and $16 \mathrm{mg} / \mathrm{bottle}$. The final concentration of acarbose in the acarbose treatment groups was $0.1,0.2$, and $0.4 \mathrm{mg} / \mathrm{mL}$, respectively. There were four replicate bottles in both the acarbose treatment groups and the control group. The four bottles containing the incubation medium without substrate and acarbose were incubated as blanks to correct the gas production resulting from the activity of the ruminal fluid.

The contents of the rumen were obtained immediately before morning feeding and squeezed through four layers of cheesecloth into a flask under $\mathrm{CO}_{2}$ in a water bath at $39^{\circ} \mathrm{C}$ until used. A $40 \mathrm{~mL}$ mixture containing $37^{\circ} \mathrm{C}$ reduced buffer medium (Theodorou et al., 1994) and strained rumen fluid in a $4: 1(\mathrm{v} / \mathrm{v})$ ratio was dispensed into each incubation bottle. Immediately after inoculation, the bottles were gassed with $\mathrm{CO}_{2}$, sealed and incubated at $39^{\circ} \mathrm{C}$ for $24 \mathrm{~h}$. Gas production was measured at 2, 4, 6, 8, 12, 16, and $24 \mathrm{~h}$ using a pressure transducer technique (Theodorou et al., 1994). At the end of the incubation, the $\mathrm{pH}$ values in each bottle were measured immediately after the bottle was uncapped, and the fermentation was then stopped by swirling the bottles on ice. The bottles were emptied into centrifuge tubes, and the solid residue remaining at the end of fermentation was separated by centrifugation at $12,000 \times \mathrm{g}$ for $10 \mathrm{~min}$. Supernatant fluid $(1 \mathrm{~mL})$ was added to $0.2 \mathrm{~mL}$ of deproteinising solution (metaphosphoric acid [100 mL/L]) for short chain fatty acids (SCFA) analysis. Another $2 \mathrm{~mL}$ of the supernatant fluid were added to $2 \mathrm{~mL}$ of $0.2 \mathrm{~mol} / \mathrm{L}-\mathrm{HCl}$ for ammonia-nitrogen $\left(\mathrm{NH}_{3}-\mathrm{N}\right)$ analysis. The remaining supernatant was used as a subsample for the analysis of lactic acid and LPS. The samples were stored at $-20^{\circ} \mathrm{C}$ for future analysis. The solid residues in the control and in the $16 \mathrm{mg}$ of acarbose/bottle treatment obtained by centrifugation were resuspended in $5 \mathrm{~mL}$ of phosphate buffer solution $\left(\mathrm{pH}\right.$ 7.2) and stored at $-80^{\circ} \mathrm{C}$ for DNA extraction.

\section{Analysis}

The SCFA concentrations were analysed according to the gas chromatography (GC-14B, Shimadzu, Japan) method proposed by Qin (1982) (capillary column. 30 $\mathrm{m} \times 0.32 \mathrm{~mm} \times 0.25 \mathrm{~mm}$ film thickness, column temperature $=110^{\circ} \mathrm{C}$, injector temperature $=180^{\circ} \mathrm{C}$ and detector temperature $\left.=180^{\circ} \mathrm{C}\right) . \quad \mathrm{NH}_{3}-\mathrm{N}$ was measured by the indophenol method (Weatherburn, 1967). The concentration 
of lactic acid was analysed following the method described by Baker and Summerson (1941). The level of LPS was measured by a chromogenic end-point Tachypleus amebocyte lysate assay kit (Chinese Horseshoe Crab Reagent Manufactory, Xiamen, China).

\section{DNA extraction and 16S rRNA gene amplicon pyrosequencing}

A total of $1 \mathrm{~mL}$ of the stored solution from the control and the $0.4 \mathrm{mg} / \mathrm{mL}$ of acarbose/bottle treatment were used for DNA extraction. The DNA was extracted by a beadbeating method using a mini-bead beater (Biospec Products Inc., Bartlesville, OK, USA), followed by phenolchloroform extraction (Mao et al., 2007). The solution was precipitated with ethanol, and the pellets were suspended in $50 \mu \mathrm{L}$ of Tris-EDTA buffer. DNA was quantified using a Nanodrop spectrophotometer (Nyxor Biotech, Paris, France), following staining using a Quant-it Pico Green dsDNA kit (Invitrogen Ltd. Paisley, UK). The DNA samples were stored at $-80^{\circ} \mathrm{C}$ until further processing.

The following universal primers were applied for the amplification of the V3-V6 region of the 16S rRNA gene: forward primer, 5'-CCATCTCATCCCTGCGTGTCTCC GACTCAGNNNNNNACTCCTACGGGAGGCAGCAG3' (the italicised sequence is a 454 Life Sciences primer A, and the bold sequence is the broadly conserved bacterial primer 338F, NNNNNN designates the sample-specific sixbase barcode used to tag each polymerase chain reaction [PCR] product), reverse primer 5'-CCTATCC CCTGTGTGCCTTGGCAGTCTCAGCRRCACGAGCTG

ACGAC-3' (the italicised sequence is 454 Life Sciences primer $\mathrm{B}$, and the bold sequence is the broadly conserved bacterial primer 1061R). The cycling parameters were as follows: $5 \mathrm{~min}$ initial denaturation at $95^{\circ} \mathrm{C}, 25$ cycles of denaturation at $95^{\circ} \mathrm{C}(30 \mathrm{~s})$, annealing at $55^{\circ} \mathrm{C}(30 \mathrm{~s})$, elongation at $72^{\circ} \mathrm{C}(30 \mathrm{~s})$ and final extension at $72^{\circ} \mathrm{C}$ for 5 min. Three separate PCR reactions of each sample were pooled for pyrosequencing. The PCR products were separated by $1 \%$ agarose gel electrophoresis and purified using a QIAquick Gel extraction kit (Qiagen, Valencia, CA, USA). Amplicons were quantified using a Quant-iT PicoGreen dsDNA Assay Kit (Invitrogen Ltd., UK). Equal concentrations of amplicons were pooled from each sample. The amplicons were sequenced as recommended by the manufacturer's instructions.

\section{Pyrosequencing data analysis}

The sequences were processed using the Mothur (version 1.29.0, April 2013) program. 16S rRNA reads were decoded based on $6 \mathrm{bp}$ sample-specific barcodes and processed to remove poor quality sequences. To reduce sequencing errors, the shhh.flows command was applied, which is the Mothur implementation of the AmpliconNoise algorithm. Quality filters were applied to trim and remove sequences with sequences less than $200 \mathrm{bp}$ in length, an average quality score less than 35 and homopolymers longer than eight nucleotides with more than two different bases than the primer. To obtain a nonredundant set of sequences, unique sequences were determined and aligned using the SILVA reference alignment database. Chimeras were removed using chimera.uchime (http.//drive5.com/ uchime). The sequences identified as being of eukaryotic origin were removed. The candidate sequences were then screened and preclustered to eliminate outliers. A distance matrix was generated from the resulting sequences. The sequences were clustered into operational taxonomic units (OTUs) using the furthest neighbour algorithm. Representative sequences from the OTUs at a 0.03 distance were obtained and classified using ribosomal database project's Bayesian classifier (Wang et al., 2007). Rarefaction curves and Good's coverage were calculated to quantify the coverage and sampling effort. Community diversity was estimated using Chaol and Shannon indices (Baker et al., 2003). The unweighted Unifrac distance method was used to perform a principal coordinates analysis (PCoA), and a distance-based molecular variance analysis (AMOVA) was conducted to assess significant differences between the samples (Good, 1953). Double hierarchical analysis was conducted using the unweighted pair-group method and Manhattan distance with no scaling using the Number Cruncher Statistical System (NCSS 2007) software (NCSS, Kaysville, UT, USA).

\section{Statistical analysis}

Fermentation data were analysed using the one-way analysis of variance program in the statistical software package SPSS vs. 17.0 (SPSS Inc., Chicago, IL, USA). Linear effects due to acarbose addition were determined using polynomial contrasts. The microbial data were analysed using the nonparametric Kruskal-Wallis test. All pvalues from the nonparametric Kruskal-Wallis test for microbial data were adjusted by the false discovery rate. Significant differences were considered at $\mathrm{p}<0.05$.

\section{RESULTS}

\section{In vitro anaerobic fermentation and gas production}

As shown in Table 1, acarbose addition linearly decreased $(\mathrm{p}<0.05)$ the gas production and the concentrations of acetate, propionate, butyrate, total short chain fatty acid (TSCFA), lactic acid and LPS, and linearly increased $(\mathrm{p}<0.05)$ the ratio of acetate to propionate, the $\mathrm{pH}$ and the concentrations of isovalerate, valerate and $\mathrm{NH}_{3}-\mathrm{N}$ compared with the control. No significant effect $(\mathrm{p}>0.05)$ 
Table 1. Effects of acarbose addition on ruminal fermentation in vitro

\begin{tabular}{|c|c|c|c|c|c|c|c|}
\hline \multirow{2}{*}{ Item } & \multicolumn{4}{|c|}{ Acarbose $(\mathrm{mg} / \mathrm{mL})$} & \multirow{2}{*}{ SEM } & \multicolumn{2}{|c|}{$\mathrm{p}$ value } \\
\hline & 0 & 0.1 & 0.2 & 0.4 & & Acarbose vs control & Linear \\
\hline$\overline{\mathrm{pH}}$ & 5.59 & 6.49 & 6.51 & 6.57 & 0.177 & $<0.001$ & $<0.001$ \\
\hline Lactic acid (mM) & 0.21 & 0.14 & 0.13 & 0.13 & 0.056 & $<0.001$ & $<0.001$ \\
\hline Acetate $(\mathrm{mM})$ & 67.94 & 61.77 & 61.88 & 62.25 & 1.437 & 0.001 & 0.001 \\
\hline Propionate (mM) & 29.24 & 18.24 & 18.04 & 18.76 & 0.539 & $<0.001$ & $<0.001$ \\
\hline Isobutyrate (mM) & 1.38 & 1.07 & 1.3 & 1.55 & 0.27 & 0.322 & 0.37 \\
\hline Butyrate (mM) & 24.74 & 8.4 & 9.24 & 8.57 & 0.453 & $<0.001$ & $<0.001$ \\
\hline Isovalerate $(\mathrm{mM})$ & 1.08 & 1.67 & 1.76 & 1.63 & 0.083 & $<0.001$ & $<0.001$ \\
\hline Valerate $(\mathrm{mM})$ & 1.5 & 1.78 & 1.86 & 1.78 & 0.067 & 0.001 & 0.001 \\
\hline Acetate to Propionate & 2.32 & 3.39 & 3.43 & 3.32 & 0.048 & $<0.001$ & $<0.001$ \\
\hline TSCFA $(\mathrm{mM})$ & 125.88 & 92.91 & 94.07 & 94.55 & 2.319 & $<0.001$ & $<0.001$ \\
\hline $\mathrm{NH}_{3}-\mathrm{N}(\mathrm{mM})$ & 5.12 & 14.36 & 16.2 & 15.41 & 0.844 & $<0.001$ & $<0.001$ \\
\hline LPS (EU/mL) & $12,965.66$ & $9,238.25$ & $7,364.5$ & $8,370.73$ & 647.702 & $<0.001$ & $<0.001$ \\
\hline Total gas production $(\mathrm{mL})$ & 155.35 & 93.58 & 90.65 & 85.95 & 3.877 & $<0.001$ & $<0.001$ \\
\hline
\end{tabular}

SEM, standard error of the mean; TSCFA, total short chain fatty acid; LPS, lipopolysaccharide; EU, endotoxin unit.

was observed in the concentration of isobutyrate between the control and the acarbose treatments.

\section{General DNA sequencing observations}

Of the pyrosequencing reads that passed the quality control tests, 18,772 were from the control group and 20,156 were from the acarbose groups. The number of sequences per sample within the two groups ranged from 4,066 in the control to 5,152 in the acarbose groups (Table 2). Overall, 14 phyla were represented in the ruminal samples (Table 3). Of the major phyla, Firmicutes (53.8\%) and Bacteroidetes $(36.3 \%)$ were the most abundant in the library. Five of the detected phyla, namely Firmicutes, Bacteroidetes, Proteobacteria, Spirochaetes, and Tenericutes, collectively constituted more than $98 \%$ of the ruminal microbiome. Each of the other phyla corresponded to less than $1 \%$ of the sequences. About $0.15 \%$ and $0.35 \%$ of the sequences could not be assigned to any bacterial phylum in the control and the acarbose group, respectively.

At the genus level, overall, a total of 68 genera belonging to the 14 phyla were observed in the control and the treatment groups. However, $35.7 \%$ of all sequences were not identified at the genus level in the present study.

Table 2. Number of reads, OTUs and coverage obtained per sample in the control and acarbose treatments

\begin{tabular}{lccc}
\hline Group & Reads & OTUs & Coverage \\
\hline Control-1 & 5,082 & 1,300 & 0.87 \\
Control-2 & 4,066 & 1,161 & 0.86 \\
Control-3 & 4,726 & 1,276 & 0.86 \\
Control-4 & 4,848 & 1,431 & 0.84 \\
Acarbose-1 & 5,092 & 1,662 & 0.84 \\
Acarbose-2 & 5,070 & 1,557 & 0.84 \\
Acarbose-3 & 5,152 & 1,486 & 0.87 \\
Acarbose-4 & 4,842 & 1,519 & 0.84 \\
\hline
\end{tabular}

OTUs, operational taxonomic units.
Among the 68 bacterial genera identified, the control and the acarbose groups were represented by 45 and 56 genera, respectively (Table 4). The most abundant genera in the control group were Prevotella (17.19\%), Pseudobutyrivibrio (11.4\%), Streptococcus (8.46\%), Ruminobacter (6.85\%), Butyrivibrio (5.65\%), and Ruminococcus (2.27\%). In the acarbose group, the most abundant genera were Prevotella (12.7\%), Streptococcus (7.36\%), Ruminococcus $(2.35 \%)$, Olivibacter $(2.17 \%)$, and Pseudobutyrivibrio (2.15\%) (Figure 1). For OTUs at the $3 \%$ distance (species level), 4,262 different phylotypes were detected among all the samples. Of these, 2,709 phylotypes were found in the control samples and 3,220 in the acarbose samples (Table 4). A Venn diagram revealed that the percentage of species-level taxa shared by the control and

Table 3. Influence of acarbose addition on the changes in the composition of ruminal bacterial microbiota at the phylum level

\begin{tabular}{|c|c|c|c|c|}
\hline \multirow[t]{2}{*}{ Phylum } & \multicolumn{2}{|c|}{$\begin{array}{c}\text { Proportion of the total } \\
\text { bacterial population }(\%)\end{array}$} & \multirow[t]{2}{*}{ SEM } & \multirow[t]{2}{*}{$\mathrm{p}$ value } \\
\hline & Control & Acarbose & & \\
\hline Firmicutes & 57.25 & 50.35 & 1.750 & 0.031 \\
\hline Bacteroidetes & 29.35 & 43.18 & 2.682 & $<0.001$ \\
\hline Proteobacteria & 9.33 & 1.1 & 1.608 & $<0.001$ \\
\hline Spirochaetes & 1.4 & 1.95 & 0.267 & 0.238 \\
\hline Tenericutes & 1.25 & 1.5 & 0.164 & 0.08 \\
\hline Cyanobacteria & 0.55 & 0.7 & 0.164 & 0.32 \\
\hline Actinobacteria & 0.35 & 0.35 & $<0.001$ & 1 \\
\hline Lentisphaerae & 0.15 & 0.08 & $<0.001$ & 0.228 \\
\hline Unclassified bacteria & 0.15 & 0.35 & $<0.001$ & 0.071 \\
\hline Synergistetes & 0.1 & 0.23 & $<0.001$ & 0.04 \\
\hline Elusimicrobia & 0.08 & 0.1 & $<0.001$ & 0.356 \\
\hline Chloroflexi & 0.03 & $<0.001$ & $<0.001$ & 0.356 \\
\hline Fibrobacteres & ND & 0.1 & $<0.001$ & 0.049 \\
\hline Verrucomicrobia & ND & 0.03 & $<0.001$ & 0.356 \\
\hline
\end{tabular}

SEM, standard error of the mean; ND, not detected. 
Table 4. Number of bacterial genera and OTUs identified in the control and acarbose treatments

\begin{tabular}{lcc}
\hline Parameters & Control & Acarbose \\
\hline Total genera & 45 & 56 \\
OTUs & 2,709 & 3,220 \\
\hline
\end{tabular}

OTUs, operational taxonomic units.

acarbose groups was 39\% (1,667 species) (Figure 2).

Influence of acarbose addition on ruminal bacterial phyla and genera in vitro

At the phylum level, as compared with the control, acarbose addition decreased $(\mathrm{p}<0.05)$ the relative abundance of Firmicutes and Proteobacteria and increased $(\mathrm{p}<0.05)$ the proportion of Bacteroidetes, Fibrobacteres and Synergistetes (Table 3). No significant effects $(p>0.05)$ were observed in the percentages of Actinobacteria, Chloroflexi, Cyanobacteria, Elusimicrobia, Lentisphaerae, Spirochaetes, Tenericutes, unclassified Bacteria, and Verrucomicrobia between the control and the acarbose group.

At the genus level, as compared with the control, acarbose addition decreased $(\mathrm{p}<0.05)$ the percentage of
Prevotella, Pseudobutyrivibrio, Ruminobacter, Butyrivibrio, Succiniclasticum, Succinatimonas, Acetitomaculum, and Solobacterium (Table 5) and increased $(\mathrm{p}<0.05)$ the percentage of Olivibacter, Xylanibacter, and Barnesiella.

Influence of acarbose addition on changes in the bacterial diversity in the rumen

Rarefaction curves indicated that acarbose addition increased the diversity of bacterial microbiota compared with the control (Figure 3). The results showed that the Shannon index and the Chao1 value of the acarbose treatment group were higher than those of the control (Figure 4). In addition, PCoA profiles revealed that the composition of the ruminal bacterial community of the acarbose and the control groups was distinct (Figure 5). The distance-based AMOVA unweighted analysis also showed that there was a significant difference in the composition of the bacterial microbiota of the control and acarbose groups $(p=0.024)$.

\section{Correlations between the fermentation data and the percentage of microbial phyla}

Pearson correlation analysis of the relative abundance of

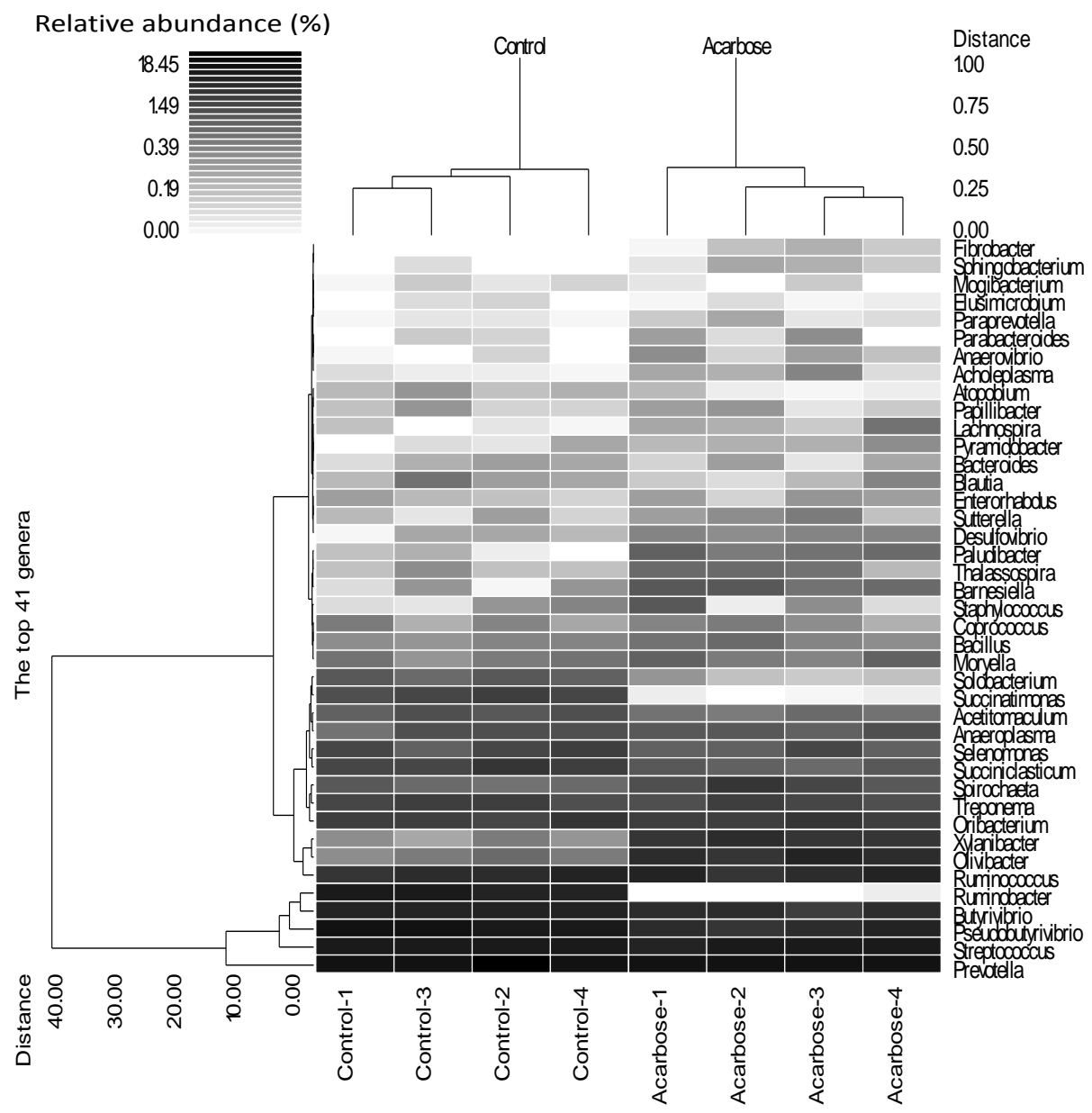

Figure 1. Influence of acarbose on the ruminal bacterial microbiota of the top 41 most abundant genera. 

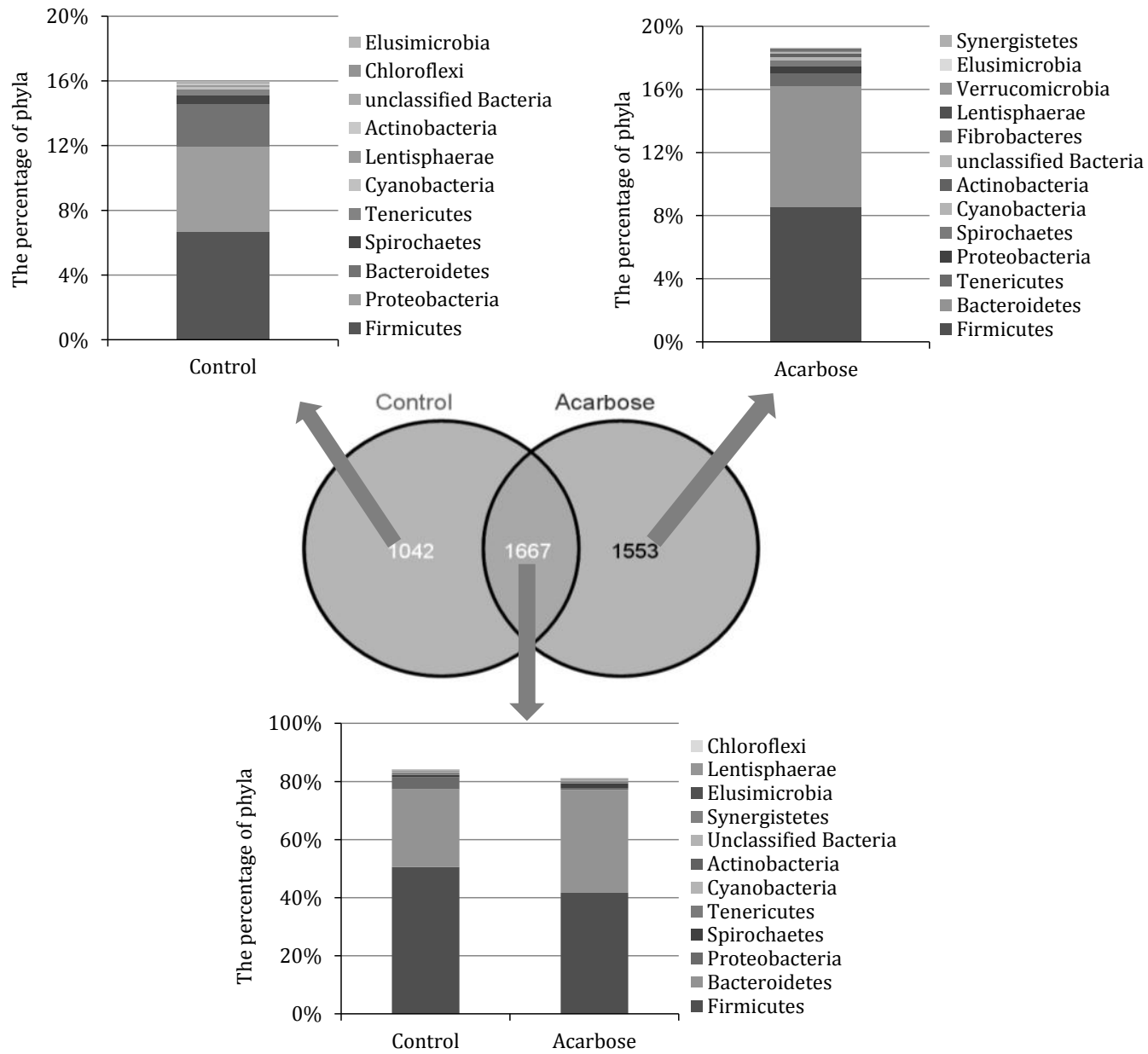

Figure 2. Venn diagram of the overlap between observed OTUs at 3\% divergence in the control and acarbose groups. OTUs, operational taxonomic units.

Table 5. Influence of acarbose on the changes in the composition of ruminal bacterial microbiota at the genus level (only the genera which relative abundance $\geq 0.5 \%$ in one group were presented)

\begin{tabular}{|c|c|c|c|c|}
\hline \multirow{2}{*}{ Genus } & \multicolumn{2}{|c|}{ Proportion of the total bacterial population (\%) } & \multirow{2}{*}{ SEM } & \multirow{2}{*}{$\mathrm{p}$ value } \\
\hline & Control & Acarbose & & \\
\hline Prevotella & 17.19 & 12.70 & 0.989 & 0.006 \\
\hline Pseudobutyrivibrio & 11.40 & 2.15 & 1.783 & $<0.001$ \\
\hline Streptococcus & 8.46 & 7.36 & 0.771 & 0.522 \\
\hline Ruminobacter & 6.85 & 0.03 & 1.351 & $<0.001$ \\
\hline Butyrivibrio & 5.65 & 1.87 & 0.730 & $<0.001$ \\
\hline Ruminococcus & 2.27 & 2.35 & 0.158 & 0.803 \\
\hline Oribacterium & 1.30 & 1.33 & 0.069 & 0.833 \\
\hline Succiniclasticum & 1.23 & 0.54 & 0.150 & 0.006 \\
\hline Selenomonas & 0.99 & 0.56 & 0.119 & 0.068 \\
\hline Treponema & 0.94 & 0.90 & 0.065 & 0.786 \\
\hline Succinatimonas & 0.92 & 0.04 & 0.175 & $<0.001$ \\
\hline Anaeroplasma & 0.65 & 0.60 & 0.050 & 0.690 \\
\hline Acetitomaculum & 0.64 & 0.35 & 0.061 & 0.002 \\
\hline Solobacterium & 0.54 & 0.14 & 0.079 & $<0.001$ \\
\hline Spirochaeta & 0.45 & 1.06 & 0.179 & 0.084 \\
\hline Olivibacter & 0.33 & 2.17 & 0.361 & $<0.001$ \\
\hline Xylanibacter & 0.24 & 1.84 & 0.307 & $<0.001$ \\
\hline Barnesiella & 0.14 & 0.50 & 0.079 & 0.006 \\
\hline
\end{tabular}

SEM, standard error of the mean. 


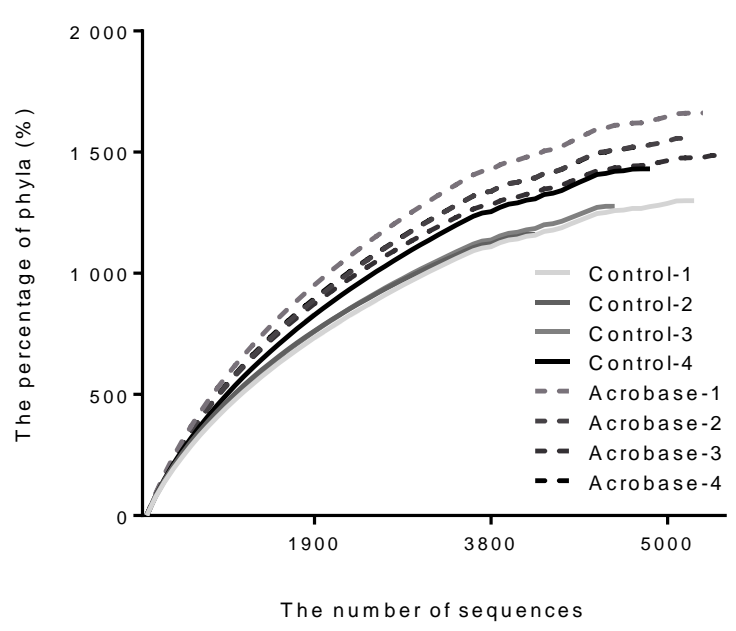

Figure 3. Summary of diversity assessments based on the operational taxonomic units (OTUs) (3\% divergence) for each sample. OTUs, operational taxonomic units.

the phyla and the ruminal fermentation data was performed (Table 6). The results showed that the phylum Bacteroidetes and Fibrobacteres were positively correlated $(\mathrm{p}<0.05)$ with ruminal $\mathrm{pH}$, the concentrations of isovalerate, valerate and $\mathrm{NH}_{3}-\mathrm{N}$ and the ratio of acetate to propionate, and negatively associated with the concentrations of LPS, lactate, acetate,
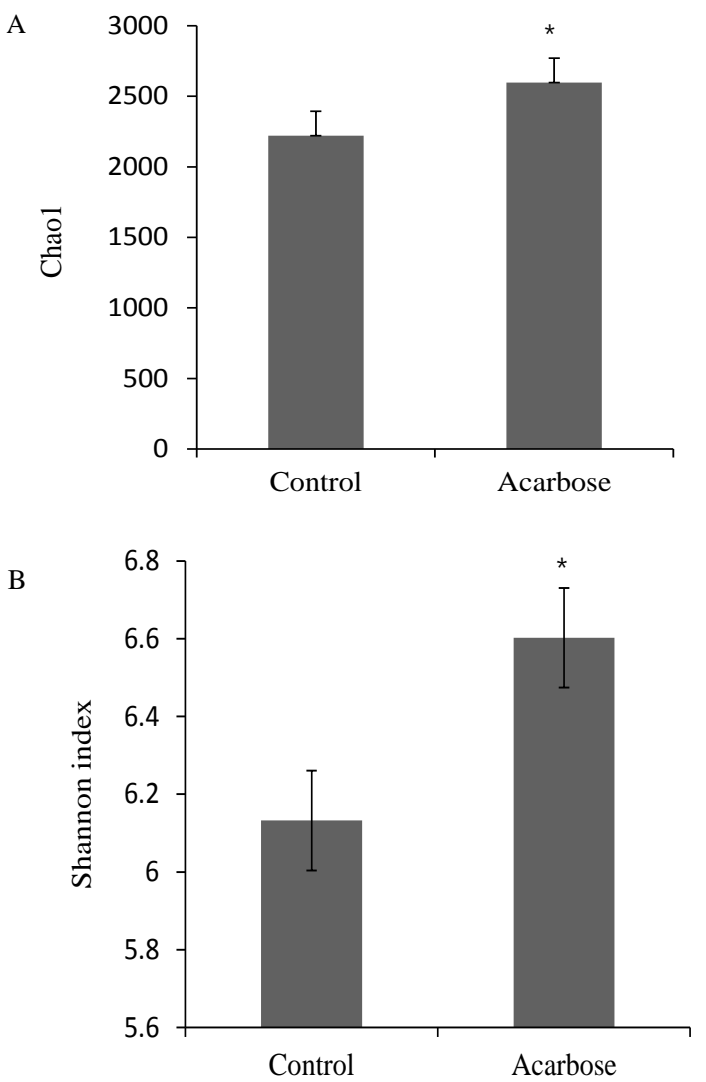

Figure 4. Influence of acarbose on the Chaol value (A) and the Shannon index of diversity (B). * $\mathrm{p}<0.05$.

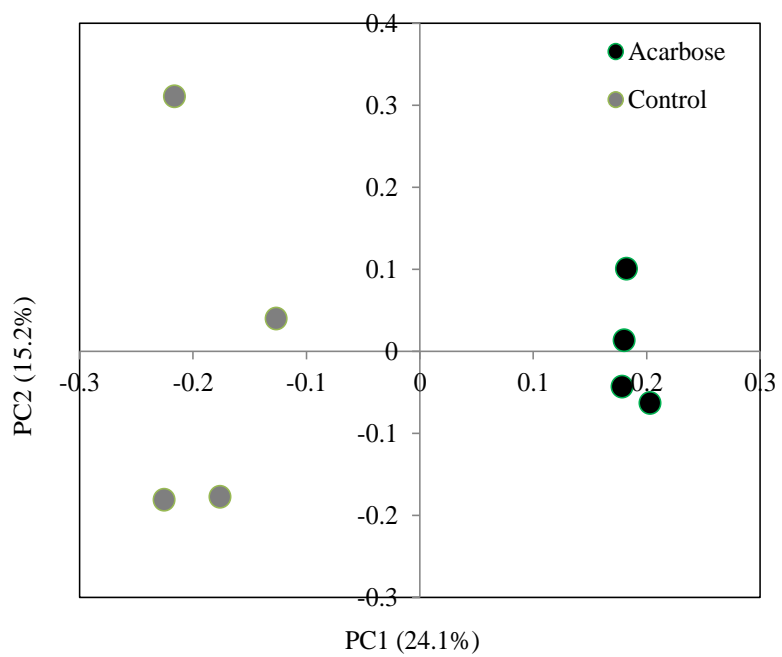

Figure 5. Results of principal coordinate analysis (PCoA) of unweighted UniFrac values of the control and acarbose group.

propionate, butyrate and TSCFA. In addition, the Firmicutes and Proteobacteria were positively correlated $(\mathrm{p}<0.05)$ with the levels of LPS, lactate, acetate, propionate, butyrate and TSCFA, and negatively correlated $(\mathrm{p}<0.05)$ with the $\mathrm{pH}$, the ratio of acetate to propionate and the concentration of $\mathrm{NH}_{3-}$ N. Another three phyla, the Synergisters, Tenericutes, and unclassified bacteria, were positively correlated with the concentration of iosvalerate. Ternericutes was also correlated with the levels of LPS and $\mathrm{NH}_{3}-\mathrm{N}$ and with the ratio of acetate to propionate.

\section{DISCUSSION}

Acarbose is a $\alpha$-amylase and glucosidase inhibitor that is indicated for the control of blood glucose in diabetic patients. The possible effect of acarbose is attributed to it decreasing starch digestion in the rumen and increasing the amount of starch digested in the intestine (Speight et al., 2007). Therefore, if a reduced amount of starch is degraded in a fermentation bottle by adding acarbose, a decrease in SCFA production is expected. McLaughlin et al. (2009a) observed a decrease in TSCFA and lactate concentrations in animals treated with acarbose compared with a control in an acute-acidosis challenge experiment in cattle. In agreement with the above report (McLaughlin et al., 2009a), the current study showed that acarbose addition increased the final $\mathrm{pH}$ and decreased the concentrations of lactate and TSCFA. However, the concentrations of $\mathrm{NH}_{3}-\mathrm{N}$, isovalerate and valerate were increased in the acarbose treatment compared with the control. It is known that the ruminal $\mathrm{NH}_{3}-\mathrm{N}$ concentration is a crude predictor of the efficiency of dietary $\mathrm{N}$ conversion to microbial $\mathrm{N}$. The increase in the $\mathrm{NH}_{3}-\mathrm{N}$ levels in the present study indicates that the addition of acarbose may have decreased the efficiency of dietary $\mathrm{N}$ conversion to microbial $\mathrm{N}$. This result implies that acarbose 
Table 6. Correlation(r) between the ruminal bacterial microbiota and the ruminal fermentation data ${ }^{1}$

\begin{tabular}{lccccccc}
\hline Variables & Bacteroidetes & Fibrobacteres & Firmicutes & Proteobacteria & Synergistetes & Tenericutes & $\begin{array}{c}\text { Unclassified } \\
\text { bacteria }\end{array}$ \\
\hline pH & $0.959^{*}$ & $0.867^{*}$ & $-0.736^{*}$ & $-0.963^{*}$ & $\mathrm{NS}$ & $\mathrm{NS}$ & $\mathrm{NS}$ \\
LPS $(\mathrm{EU} / \mathrm{mL})$ & $-0.917^{*}$ & $-0.75^{*}$ & $0.742^{*}$ & $0.9^{*}$ & $\mathrm{NS}$ & $-0.86^{*}$ & $\mathrm{NS}$ \\
Lactate $(\mathrm{mM})$ & $-0.956^{*}$ & $-0.879^{*}$ & $0.725^{*}$ & $0.973^{*}$ & $\mathrm{NS}$ & $\mathrm{NS}$ & $\mathrm{NS}$ \\
Acetate $(\mathrm{mM})$ & $-0.921^{*}$ & $-0.752^{*}$ & $\mathrm{NS}$ & $0.928^{*}$ & $\mathrm{NS}$ & $\mathrm{NS}$ & $\mathrm{NS}$ \\
Propionate $(\mathrm{mM})$ & $-0.962^{*}$ & $-0.844^{*}$ & $0.734^{*}$ & $0.975^{*}$ & $\mathrm{NS}$ & $\mathrm{NS}$ & $\mathrm{NS}$ \\
Butyrate $(\mathrm{mM})$ & $-0.957^{*}$ & $-0.869^{*}$ & $0.727^{*}$ & $0.973^{*}$ & $\mathrm{NS}$ & $\mathrm{NS}$ & $\mathrm{NS}$ \\
Isovalerate $(\mathrm{mM})$ & $0.903^{*}$ & $0.896^{*}$ & $\mathrm{NS}$ & $-0.971^{*}$ & $0.752^{*}$ & $0.726^{*}$ & $0.791^{*}$ \\
Valerate $(\mathrm{mM})$ & $0.761^{*}$ & $0.727^{*}$ & $\mathrm{NS}$ & $-0.875^{*}$ & $\mathrm{NS}$ & $\mathrm{NS}$ & $\mathrm{NS}$ \\
TSCFA $(\mathrm{mM})$ & $-0.961^{*}$ & $-0.838^{*}$ & $0.733^{*}$ & $0.972^{*}$ & $\mathrm{NS}$ & $\mathrm{NS}$ & $\mathrm{NS}$ \\
A:P & $0.967^{*}$ & $0.847^{*}$ & $-0.752^{*}$ & $-0.967^{*}$ & $\mathrm{NS}$ & $0.717^{*}$ & $\mathrm{NS}$ \\
NH $\mathrm{H}_{3} \mathrm{~N}(\mathrm{mM})$ & $0.988^{*}$ & $0.869^{*}$ & $-0.844^{*}$ & $-0.926^{*}$ & $\mathrm{NS}$ & $0.728^{*}$ & $\mathrm{NS}$ \\
\hline $\mathrm{NS}, \mathrm{ns}$
\end{tabular}

NS, not significant; LPS, lipopolysaccharide; EU, endotoxin unit; TSCFA, total short chain fatty acid; A:P, the ration of acetate to propionate.

${ }^{1}$ Only the phyla whose abundance was significantly associated with fermentation variables are presented.

$* \mathrm{p}<0.05$.

addition may affect the production of microbial protein.

There is limited information on the effect of acarbose addition on the composition of the ruminal community. Blanch et al. (2010) reported that acarbose addition (0.75 $\mathrm{g} / \mathrm{d}$ ) decreased the Streptococcus bovis to Megasphaera elsdenii ratio in the rumen of dairy cattle fed a high grain diet compared with the control group, suggesting that acarbose may affect the composition of ruminal microflora. In this study, a pyrophosphate sequencing approach was adopted to monitor the change in the composition of the ruminal bacterial community. The results revealed that acarbose addition resulted in an increased diversity, with the predicted Chaol values and Shannon index much higher in the acarbose treatment group compared to the control group (Figure 4). In addition, PCoA profiles also revealed that acarbose addition altered the composition of the ruminal bacterial community (Figure 5). A plausible explanation for these findings is that the activity of $\alpha$-amylase/glucosidase produced by the members of the rumen bacterial microbiota may be inhibited in the rumen, and the inhibition of $\alpha$ amylase/glucosidase activity reduced the rate of degradation of starch to glucose and thus the supply of glucose available for SCFA production should be reduced. The decrease in the supply of glucose may result in lower energy production and further reduce the growth rates of ruminal bacteria, and finally alter the composition and structure of rumen bacterial community.

Proteobacteria are predominantly composed of gramnegative bacteria, which have highly diverse metabolic functions (Nagaraja and Titgemeyer, 2007), especially in ruminal $\mathrm{N}$ metabolism. The decreased detection of Proteobacteria in the acarbose treatment group suggests a decrease in the numbers of bacterial species that are metabolically capable of handling the protein content of the substrate (Table 3). Firmicutes were abundant in the $16 \mathrm{~S}$
rRNA libraries of both groups. Firmicutes are mainly comprised of gram-positive, low $\mathrm{G}+\mathrm{C}$-content bacteria (Boone et al., 2001). Thus, the presence of Firmicutes in high abundance in both groups suggests that Firmicutes represent a core bacterial component within the rumen. In addition, at the phylum level for the bacteria detected in this study, acarbose addition increased the percentage of Bacteroidetes, whereas Firmicutes was less abundant in the acarbose treatment than in the control. As mentioned earlier, Firmicutes are a core bacterial component within the rumen. Thus, the decreased detection of Firmicutes following acarbose treatment suggests a decrease in the abundance of bacterial species that are metabolically capable of handing the whole substrate. In line with this idea, in the present study, a lower concentration of TSCFA was observed in the acarbose group compared with the control. Bacteroidetes are the most abundant gram-negative bacteria found in the anaerobic communities of the rumen. The decrease in the abundance of Bacteroidetes may be partly explained by the low final $\mathrm{pH}$ in the control group, as a low rumen $\mathrm{pH}$ can result in the death and lysis of gram-negative bacteria (Russell and Diez, 1998). The libraries from the acarbose group contained significantly higher numbers of bacteria belonging to the phylum Fibrobacteres (Table 3). Fibrobacter spp. have been identified as the major cellulolytic bacteria species present within the rumen (Denman and McSweeney, 2006). In the present study, this observation of Fibrobacter species in the acarbose treatment group indicates that acarbose may have little negative effects on the fermentation of Fibrobacter species.

This study also detected a significant difference in the population structure at the genus level between the control and the acarbose groups. We observed that the proportions of the predominant genera, including Prevotella, Pseudobutyrivibrio, Ruminobacter, Butyrivibrio, and 
Succiniclasticum, were reduced in the acarbose groups. Among the predominant genera whose proportions were decreased, the Prevotella genus is reported to be the most predominant in the rumen (Mao et al., 2013). In the present study, the Prevotella genus was more abundant in the control group than in the acarbose groups (Table 5), suggesting that the growth of Prevotella spp. may be inhibited by the addition of acarbose. In the rumen, amylase can be produced by the members of the Butyrivibrio/Pseudobutyrivibrio (Moon et al., 2008). The current study showed that acarbose addition led to a decrease in the abundance of Pseudobutyrivibrio and Butyrivibrio. A possible explanation for this finding could be that acarbose effectively limited the availability of starch to the members of these genera listed above, and thus decreased the growth of Pseudobutyrivibrio sp. and Butyrivibrio sp. On the other hand, this study also showed that the proportions of Olivibacter and Xylanibacter were significantly increased in the acarbose group when compared with the control group. One possible explanation for this finding may be that carbohydrate metabolisms of members of these genera mentioned earlier were not affected by acarbose.

Previous studies have shown that feeding high levels of grain to dairy cows leads to a decline in ruminal $\mathrm{pH}$ (Denman and McSweeney, 2006; Plaizier et al., 2008), resulting in the death and cell lysis of gram-negative bacteria and a subsequent increase in the concentration of free LPS in the rumen (Plaizier et al., 2008). In addition, previous studies showed that rapid growth of gram-negative bacteria can result in the shedding of LPS in the rumen (Plaizier et al., 2008). In agreement with these studies, the present study detected a lower ruminal $\mathrm{pH}$ and a higher concentration of LPS in the control compared with the acarbose group. The present study also demonstrated that acarbose altered the composition of the ruminal bacterial microbiota, with a decreased abundance of Firmicutes (gram-positive species) and Proteobacteria (gram-negative species) among the total number of sequences and an increased abundance of Bacteroidetes and Fibrobacteres (gram-negative species). In addition, our results revealed a direct negative correlation between the LPS level and the abundance of some gram-negative species, including Bacteroidetes spp. and Fibrobacteres spp., and a positive correlation with the percentage of Proteobacteria spp. (Table 6). Therefore, our study indicates that the higher free LPS levels in the control may be due to the death and cell lysis of Bacteroidetes and Fibrobacteres and the rapid growth of Proteobacteria spp. In addition, these findings suggest that it is possible to prevent the accumulation of LPS by acarbose addition.

As mentioned earlier, our results revealed that acarbose addition increased the ruminal $\mathrm{pH}$ and decreased the concentrations of acetate, propionate and TSCFA; however, it is noteworthy that the level of acarbose used in the present study is very high compared with the doses reported in vivo trials. For instance, Blanch et al (2010) fed the acarbose to the animals at $0.75 \mathrm{~g} / \mathrm{cow} / \mathrm{d}$, as prediction of a $20 \mathrm{~kg}$ intake. In the present study, the level of acarbose was 0.1 to $0.4 \mathrm{mg} / \mathrm{mL}$ containing $1 \mathrm{~g}$ of substrates, and this amount corresponds to 2 to $8 \mathrm{~g} / 20 \mathrm{~kg}$ of diets. Therefore, despite the fact that obvious fermentation inhibition was detected in the present study with dose dependent manner, it should be pointed out that the high dose level may result in a negative effect on in vitro fermentation, as that high levels of acarbose in the rumen appears to be involved, not only in inhibiting carbohydrate degradation in the rumen environment, but decreasing the activities (i.e. rate of microbial growth) and numbers of rumen bacterial microbiota, which might decrease the efficiency of microbial protein synthesis. Indeed, the present study showed that there was a higher concentration of $\mathrm{NH}_{3}-\mathrm{N}$ in the acarbose group compared with the control, indicating that the addition of acarbose may have decreased the efficiency of dietary $\mathrm{N}$ conversion to microbial $\mathrm{N}$. These findings also imply that acarbose may affect the microbial protein production and have a negative impact on animal performance if the dose was excessive.

\section{CONCLUSION}

It can be concluded that acarbose has a beneficial effect on ruminal fermentation of high-concentrate feeds by increasing the final $\mathrm{pH}$ and reducing LPS levels and TSCFA production, especially the concentration of butyrate. The pyrosequencing analysis showed that acarbose can increase the diversity of the ruminal bacterial community and decrease the proportion of butyrate-producing bacteria in the rumen. Thus, acarbose has potential for use as a feed additive in manipulating ruminal fermentation in ruminants.

\section{ACKNOWLEDGMENTS}

The present study was supported by the National Key Basic Research Program of China (2011CB100801).

\section{REFERENCES}

Baker, G., J. J. Smith, and D. A. Cowan. 2003. Review and reanalysis of domain-specific $16 \mathrm{~S}$ primers. J. Microbiol. Methods 55:541-555.

Baker, S. B. and W. H. Summerson. 1941. The colorimetric determination of lactic acid in biological material. J. Biol. Chem. 138:535-554.

Blanch, M., S. Calsamiglia, M. Devant, and A. Bach. 2010. Effects 
of acarbose on ruminal fermentation, blood metabolites and microbial profile involved in ruminal acidosis in lactating cows fed a high-carbohydrate ration. J. Dairy Res. 77:123-128.

Boone, D. R., R. W. Castenholz, and G. M. Garrity. 2001. Bergey's manual of systematic bacteriology. 2nd Ed. Springer. New York, NY, USA.

Cook, N. B., K. V. Nordlund, and G. R. Oetzel. 2004. Environmental influences on claw horn lesions associated with laminitis and subacute ruminal acidosis in dairy cows. J. Dairy Sci. 87(Supp. 1): E36-E46.

Denman, S. E. and C. S. McSweeney, 2006. Development of a real-time PCR assay for monitoring anaerobic fungal and cellulolytic bacterial populations within the rumen. FEMS Microbiol. Ecol. 58:572-582.

Desnoyers, M., R. S. Giger-Reverdin, G. Bertin, C. DuvauxPonter, and D. Sauvant. 2009. Meta-analysis of the influence of Saccharomyces cerevisiae supplementation on ruminal parameters and milk production of ruminants. J. Dairy Sci. 92:1620-1632.

Garrett, E. F., K. V. Nordlund, W. J. Goodger, and G. R. Oetzel. 1997. A cross-sectional field study investigating the effect of periparturient dietary management on ruminal $\mathrm{pH}$ in early lactation dairy cows. J. Dairy Sci. 80(Suppl. 1):169 (Abstr).

Good, I. L. 1953. The population frequencies of species and the estimation of population parameters. Biometrika 40(3-4):237264.

Krause, K. M. and G. R. Oetzel. 2006. Understanding and preventing subacute ruminal acidosis in dairy herds. A review. Anim. Feed Sci. Technol. 126:215-236.

Mao, S. Y., G. Zhang, and W. Y. Zhu. 2007. Effect of disodium fumarate on in vitro rumen fermentation of different substrates and rumen bacterial communities as revealed by denaturing gradient gel electrophoresis analysis of $16 \mathrm{~S}$ ribosomal DNA. Asian Australas. J. Anim. Sci. 20:543-549.

Mao, S. Y., R. Y. Zhang, D. S. Wang, and W. Y. Zhu. 2013. Impact of subacute ruminal acidosis (SARA) adaptation on rumen microbiota in dairy cattle using pyrosequencing. Anaerobe 24: 12-19.

McLaughlin, C. L., A. Thompson, K. Greenwood, J. Sherington, and C. Bruce. 2009a. Effect of acarbose on acute acidosis. J. Dairy Sci. 92:2758-2766.

McLaughlin, C. L., A. Thompson, K. Greenwood, J. Sherington, and C. Bruce. 2009b. Effect of acarbose on milk yield and composition in early-lactation dairy cattle fed a ration to induce subacute ruminal acidosis. J. Dairy Sci. 92:4481-4488.
Moon, C. D., D. M. Pacheco, W. J. Kelly, S. C. Leahy, D. Li, J. Kopečný, and G. T. Attwood. 2008. Reclassification of Clostridium proteoclasticum as Butyrivibrio proteoclasticus comb. nov., a butyrate-producing ruminal bacterium. Int. J. Syst. Evol. Microbiol. 58:2041-2045.

Nagaraja, T. G., E. E. Bartley, L. R. Fina, and H. D. Anthony. 1978. Relationship of rumen gram-negative bacteria and free endotoxin to lactic acidosis in cattle. J. Anim. Sci. 47:13291336.

Nagaraja, T. G. and E. C. Titgemeyer. 2007. Ruminal acidosis in beef cattle: the current microbiological and nutritional outlook. J. Dairy Sci. 90(Supp. 1):E17-E38.

Packer, E. L., E. H. Clayton, and P. M. V. Cusack. 2011. Rumen fermentation and live weight gain in beef cattle treated with monensin and grazing lush forage. Aust. Vet. J. 89:338-345.

Plaizier, J. C., D. O. Krause, G. N. Gozho, and B. W. McBride. 2008. Subacute ruminal acidosis in dairy cows: The physiological causes, incidence and consequences. Vet. J. 176: 21-31.

Qin, W. L. 1982. Determination of rumen volatile fatty acids by means of gas chromatography. J. NJ Agric. Col. 4:110-116.

Remling, N., S. Riede, P. Lebzien, U. Meyer, M. Höltershinken, S. Kersten, and S. Dänicke. 2013. Effects of fumaric acid on rumen fermentation, milk composition and metabolic parameters in lactating cows. J. Anim. Physiol. Anim. Nutr. (In press).

Russell, J. B. and G. F. Diez. 1997. The effects of fermentation acids on bacterial growth. Adv. Microb. Physiol. 39:205-234.

Speight, S. M., D. L. Harmon, and J. M. Tricarico. 2007. Application of carbohydrase inhibitors to moderate rumen fermentation: continuous culture evaluation. J. Dairy Sci. 90(Suppl. 1):340 (Abstr).

Theodorou, M. K., B. A. Williams, M. S. Dhanoa, A. B. McAllan, and J. France. 1994. A simple gas production method using a pressure transducer to determine the fermentation kinetics of ruminant feeds. Anim. Feed Sci. Technol. 48:185-197.

Wang, Q., G. M. Garrity, J. M. Tiedje, and J. R. Cole. 2007. Naive Bayesian classifier for rapid assignment of rRNA sequences into the new bacterial taxonomy. Appl. Envitron. Microbiol. 73:5261-5267.

Weathburn, M. W. 1967. Phenol-Hypochlorite reaction for determination of ammonia. Anal. Chem. 39:971-974.

Zebeli, Q. and B. U. Metzler-Zebeli. 2012. Interplay between rumen digestive disorders and diet-induced in flammation in dairy cattle. Res. Vet. Sci. 93:1099-1108. 